CLINICAL STUDY

\title{
Complex relationship between free thyroxine and TSH in the regulation of thyroid function
}

\author{
Rudolf Hoermann $^{1}$, Walter Eckl ${ }^{1}$, Christian Hoermann ${ }^{1}$ and Rolf Larisch ${ }^{2}$ \\ Departments of ${ }^{1}$ General Internal Medicine, Gastroenterology and Endocrinology and ${ }^{2}$ Nuclear Medicine, Klinikum Luedenscheid, Paulmannshöher Straße \\ 14, D-58515 Luedenscheid, Germany \\ (Correspondence should be addressed to R Hoermann; Email: rudolf.hoermann@gmail.com)
}

\begin{abstract}
Objective: The present study re-evaluates the inverse log TSH-free thyroxine $\left(\mathrm{fT}_{4}\right)$ relationship, which has generally been assumed to characterize the thyroid pituitary hypothalamic feedback regulation in thyroid function.

Design and Methods: The correlation between $\mathrm{fT}_{4}$ and TSH was analyzed in two data sets from differing time periods involving 3223 and 6605 patients referred for thyroid testing, representing the whole range of thyroid functions from hypothyroidism to hyperthyroidism.

Results: We found that the data do not support a linear log TSH-fT 4 relationship; instead, the correlation's gradient varies with thyroid function. As a consequence, an alternate model, based on the error function, was introduced. When directly comparing the models by means of curve fitting, using $F$-test and Akaike criteria, the alternate model results in a significantly better fit. The model was verified in the independent second set of data. Subgroup analysis of untreated patients added further proof to the non-linear model.

Conclusions: We propose a refined non-linear model to describe the relationship between TSH and fT $\mathrm{f}_{4}$. It implies that TSH response to a deviating $\mathrm{fT}_{4}$ value may not be log-linear, but may be disproportionally related to the extent of the deviation from an optimum set point. A better understanding of the complex nature of the $\mathrm{TSH}-\mathrm{fT}_{4}$ relationship may further the development of more precise clinical models and aid in better defining subclinical states of thyroid dysfunction. Also, it may encourage other biological interrelations to be reconsidered in the wake of advanced measurement techniques and more powerful computerized statistical procedures.
\end{abstract}

European Journal of Endocrinology 162 1123-1129

\section{Introduction}

Thyroid hormones are critical to energy homoeostasis in humans, and are tightly controlled. Regulation is mainly achieved by a negative thyroid pituitary hypothalamic feedback loop. This results in an increase in the secretion of TSH by the pituitary gland in case of a decline in free thyroid hormone levels in the circulation owing to a reduced hormone production by the thyroid gland, and, on the other hand, a diminished pituitary TSH response in the presence of an increased hormone output and rising circulatory thyroid hormones (1).

The understanding of the regulation of thyroid function during the last decades has fostered the development of extremely sensitive and accurate thyroid function tests, relying primarily on the determination of TSH (2). The determination of TSH, as opposed to the direct measurement of thyroid hormones, as a first-line approach to reveal an abnormal thyroid function in a patient, permits diagnosis of more subtle states of thyroid failure termed subclinical hyperthyroidism or subclinical hypothyroidism. These two conditions are characterized by a diminished and elevated TSH respectively despite free thyroxine $\left(\mathrm{fT}_{4}\right)$ and free tri-iodothyronine $\left(\mathrm{fT}_{3}\right)$ values that are still within the reference range (3).

Recently, the reference range of TSH has become a matter of debate that is not yet resolved (4-6). The issue seems to be relevant, because a minor change in the reference range of TSH would result in a considerably larger portion of patients to be judged hyper- or hypothyroid in a clinical setting $(7,8)$.

In this respect, most studies have focused on various aspects of TSH measurement, whereas the relationship between TSH and $\mathrm{fT}_{4}$ has received scant attention. TSH has generally been assumed to be inversely loglinearly related to $\mathrm{fT}_{4}$, based mainly on a few earlier studies $(1,9-11)$.

In the present study, we aim to revisit and redefine the relationship between $\mathrm{fT}_{4}$ and TSH in the light of more refined assay techniques and advanced computerized statistical methods that have become available. 


\section{Materials and methods}

\section{Patients and data collection}

Data from thyroid function testing were obtained from the laboratory database of the Department of Nuclear Medicine. The first sampling period, resulting in data set 1, was from July 2004 to January 2005 and included 4028 thyroid function tests from 4028 adults, in-patients or out-patients (median age 55 years, range 18-100 years), which had been referred to the Departments of Endocrinology or Nuclear Medicine of Klinikum Luedenscheid for suspicion or treatment of various thyroid diseases, including benign diffuse and nodular goiter, Graves' disease, thyroid autonomy, autoimmune and non-immunogenic thyroiditis and thyroid malignancies. For verification, a second independently collected set of data, which will be referred to as data set 2, was obtained from October 2006 to January 2007 involving 7629 patients (median age 62 years, range $18-98$ years).

The data $(n=780$ and 1024 in data set 1 and data set 2 respectively) from patients with pituitary hypothalamic disorders (e.g. secondary hypothyroidism) or other known conditions in which thyroid pituitary regulation may be severely compromised, such as critically ill patients including patients with renal failure, were excluded before analyses were performed, as were pregnant women and all paediatric subjects below the age of 18. Also, a few extreme values or outliners $(n=25)$ were excluded for statistical purposes, as indicated in the legend of Fig. 1.

We analyzed the data from the two sets including 3223 (1787 ambulatory and 1436 hospitalized patients) and 6605 patients (3984 ambulatory and 2621 hospitalized patients) on various thyroid medications, mainly $\mathrm{L}_{-} \mathrm{T}_{4}(n=441$ and 1092 respectively), iodine $\left(n=24\right.$ and 71), combination of iodine and $\mathrm{L}-\mathrm{T}_{4}$ $(n=50$ and 120) or antithyroid drugs $(n=40$ and 87$)$ and, in addition, the subgroups of untreated patients ( $n=709$ in data set 1 and 1356 in data set 2) receiving no thyroid medication or other drugs.

Distribution of $\mathrm{fT}_{4}$ and TSH values in data set 1 is shown in Fig. 1, Panel A.

All thyroid function tests performed were analyzed by a single institution, the Nuclear Medicine Laboratory of the hospital. Klinikum Luedenscheid is a teaching hospital of the University of Bonn. Luedenscheid is located in an area of mild iodine deficiency.

\section{Laboratory methods}

Laboratory evaluation included measurement of $\mathrm{fT}_{4}, \mathrm{fT}_{3}$ and TSH. In addition, thyroid autoantibodies (thyroid peroxidase antibodies (TPO Abs) or TSH receptor antibodies (TSH-R Abs)) were determined in case of suspicion or for the purpose of exclusion of thyroid autoimmune disorders. Results were positive in 284 patients for TPO Abs and in 105 patients for TSH-R Abs in data set 2. Documentation was insufficient for data set 1 .

TSH was measured with an enzyme immunoassay (Abbott). The working range for this method is 0.01-100 mIU/l. The reference (normal) range for the test is $0.4-4.1 \mathrm{mIU} / \mathrm{l}$. $\mathrm{fT}_{3}$ and $\mathrm{fT}_{4}$ were determined by the same method, with the reference range being 9.5-25 pmol/l for serum $\mathrm{fT}_{4}$ and $3.5-8.5 \mathrm{pmol} / \mathrm{l}$ for serum $\mathrm{fT}_{3}$.

TPO Abs were determined by RIA (reference range $<60 \mathrm{IU} / \mathrm{ml}$ ) and TSH-R Abs by the second-generation TRAK assay (reference range $<2$ U/l; BRAHMS, Berlin, Germany).

Standard laboratory quality evaluation procedures were routinely employed, and regular participation at inter-laboratory tests was also part of the quality management strategy.

\section{Statistical methods}

Spreadsheet functions and initial basic statistical analyses, such as data transformation and calculation and graphing of the $\log \mathrm{TSH}-\mathrm{fT}_{4}$ linear regression, were performed on a PC using Excel basic edition 2003, Microsoft Corp. For more advanced statistical purposes, SPSS version 15.0 (SPSS Inc., Chicago, IL, USA) was used. Linear and non-linear curve fitting and advanced statistical comparisons ( $F$-test and Akaike criteria) of the linear and non-linear fitting models were carried out using GraphPad Prism version 5.0 for Mac and Windows, GraphPad Software, San Diego, CA, USA (12).

The two models to be compared were the linear correlation $\log \mathrm{TSH}_{-} \mathrm{fT}_{4}$ (function 1) and a non-linear correlation based on the error function (erf; function 2) reflecting an increasingly stronger counteraction depending on the deviation from a putative optimum:

function $1: \log \mathrm{TSH}=a \times \mathrm{fT}_{4}+b$,

function $2: \log \mathrm{TSH}$

$$
\begin{aligned}
= & \pi^{0.5} \times k /(2 \times q) \times \operatorname{erf}(q \times(x-a))+d \times(x \\
& -a)+b .
\end{aligned}
$$

\section{Results}

Distribution of TSH and $\mathrm{fT}_{4}$ values for data set 1 and a linear fit of $\log \mathrm{TSH}-\mathrm{fT}_{4}$, considering the whole range of values and three distinct segments of the spectrum, are shown in Fig. 1. The regression lines of the three segments are significantly different from each other and from the regression line of the total data (Fig. 1). The regression line in the middle segment is flat, 

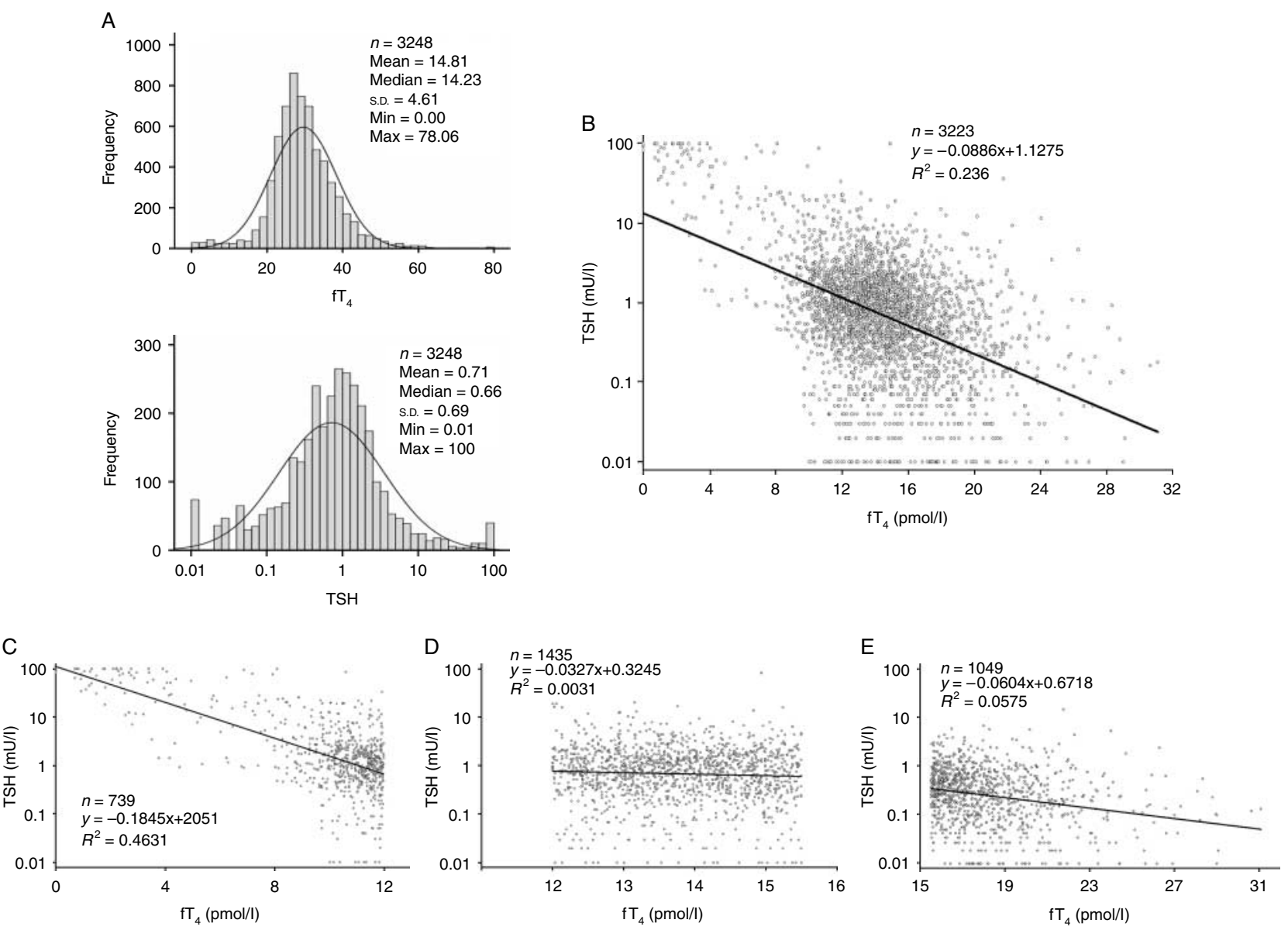

Figure 1 Distribution of $\mathrm{fT}_{4}$ and TSH values and plots of $\mathrm{fT}_{4}$ versus log TSH. Panel A shows the distribution of $\mathrm{fT}_{4}$ and $\mathrm{TSH}$ values in data set 1. The topmost graph of $\mathrm{fT}_{4}$ versus log TSH (B) depicts the whole range of data, whereas the graphs below (C-E) show the data split across three distinct segments of $\mathrm{fT}_{4}$ values. The respective lines represent the linear regression fitted to the data points with the use of Excel. The equations are shown within the respective figures. Please note the differing slopes of the lines among the various graphs. A few extreme $\mathrm{fT}_{4}$ values $(n=25)$ were not considered for the calculations depicted in these figures.

but neighbouring segments in the hypothyroid and hyperthyroid range show an ascending or descending behaviour, suggesting that a simple model with a straight line representing the $\log \mathrm{TSH}_{-} \mathrm{fT}_{4}$ inverse relationship may not be appropriate to fit the data over the whole range from hyperthyroidism to hypothyroidism.

The emerging model, consisting of at least three distinct segments, was further evaluated with the use of SPSS statistics regression tool. The analysis confirmed the differences in the slopes of regression lines over the course of the data points to be significant (Fig. 2).

Next, a new model based on function 2 was introduced. It provided a superior fit to data set 1 compared with the linear regression (function 1), as evidenced by means of comparative curve fitting carried out with Graphpad Prism including F-test and Akaike criteria (Fig. 3). Initial estimates of the constants were entered on the basis of Excel and SPSS plots depicted in
Figs 1 and 2. The following best-fit values were obtained for function $1, y$-intercept 1.10 , slope -0.09 ; s.E.M.: $y$-intercept 0.04 , slope $0.003 ; 95 \%$ confidence intervals (CI): $y$-intercept $1.02-1.18$, slope 0.09 to -0.08 ; goodness of fit: degrees of freedom 3221, $R^{2} 0.30$, absolute sum of squares 1187, $s_{y . x} 0.61$, and, for function $2, k 0.18, q 0.09, a 18.00, d-0.22, b-0.39$; S.E.M.: $k 0.047 q 0.027, d 0.052, b 0.016$; $95 \%$ CI: $k 0.08$ to $0.27 q 0.04$ to $0.14, d-0.32$ to $-0.12, b-0.42$ to -0.36 ; goodness of fit: degrees of freedom 3219, $R^{2} 0.26$, absolute sum of squares $1138, s_{y \cdot x} 0.59$.

Function 2 has next been validated on a second independent set of data obtained from a later sampling period. Curve fitting analysis to data set 2 was performed yielding the following best-fit values for function $1, y$-intercept 1.13 , slope -0.09 ; s.E.M.: $y$-intercept 0.03 , slope $0.002 ; 95 \%$ CI: $y$-intercept 1.08 to 1.18 , slope -0.093 to -0.086 ; goodness of fit: degrees of freedom 6603, $R^{2} 0.30$, absolute sum 


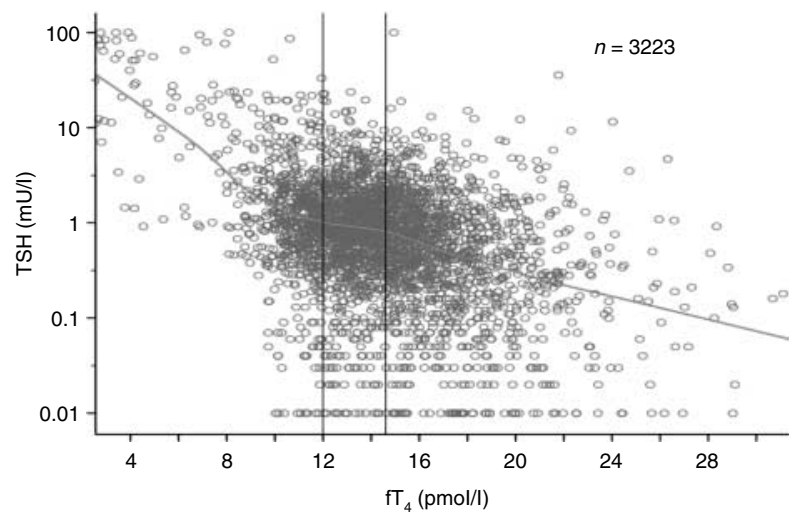

Figure 2 Regression analysis of $\mathrm{fT}_{4}$ versus log TSH. Fitting data set 1 with the use of SPSS and cut-off values estimated from Fig. 1 indicated three distinct regression lines, which were significantly different from each other and described by the following equations: a $\left(\mathrm{fT}_{4}<12\right): \mathrm{TSH}=-0.13 \times \mathrm{fT}_{4}+1.46$; b $\left(12 \leq \mathrm{fT}_{4} \geq 15\right): \mathrm{TSH}=-0.06 \times \mathrm{fT}_{4}+0.05 ; \mathrm{c}\left(\mathrm{fT}_{4}>15\right): \mathrm{TSH}$ $=-0.07 \times \mathrm{fT}_{4}+0.89$. a versus $\mathrm{b}: P<0.0001 ; \mathrm{b}$ versus c: $P=0.0002$; and a versus c: $P=0.0001$.

of squares $2166, s_{y \cdot x} 0.57$, and, function $2, k 0.24$, $q 0.066, a 17.50, d-0.30, b-0.39$; s.E.M.: $k 0.12$, $q 0.025, d 0.13, b 0.01 ; 95 \%$ CI: $k-0.04$ to $0.48, q$ -0.11 to $-0.02, d-0.54$ to $-0.05, b-0.41$ to -0.37 ; goodness of fit: degrees of freedom $6601, R^{2}$ 0.32 , absolute sum of squares $2089, s_{y \cdot x} 0.56$. When analysing the subgroup of untreated patients, results were as follows: for function $1, y$-intercept 1.18 , slope -0.09; s.E.M.: $y$-intercept 0.05 , slope $0.004 ; 95 \%$ CI: $y$-intercept $1.07-1.28$, slope -0.10 to -0.09 ; goodness of fit: degrees of freedom $1354, R^{2} 0.31$, absolute sum of squares $279, s_{y . x} 0.45$, and, function $2, k 0.14$, $q 0.14, a 18.00, d-0.18, b-0.39$; s.Е.M.: $k 0.02$,

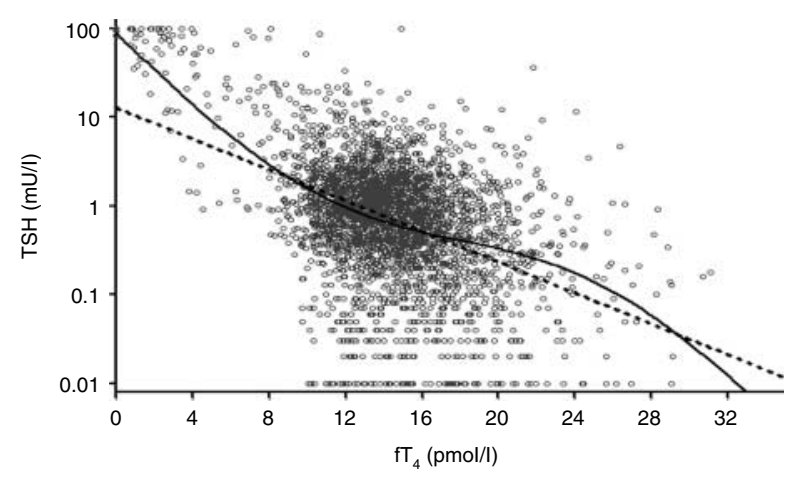

Figure 3 Comparative curve fitting applying function 1 and function 2 to data set 1 . Curve fitting was performed using Graphpad Prism to directly compare function 2 (solid line) and linear relation (function 1, broken line). Best-fit values were reported in the results section. Function 2 provided a significantly better fit to the data. Statistical results were as follows: probability null hypothesis function 1 is correct $0.01 \%$, alternative hypothesis function 2 $99.99 \%$, preferred model function 2 , differences in AICc 133.06, F:Dfn/Dfd 96.92/2.32, $P$ value $<0.0001$, conclusion $(\alpha=0.05)$ reject null hypothesis, $n=3223$. $q 0.03, d 0.03, b 0.03 ; 95 \%$ CI: $k 0.10$ to $0.19, q 0.07$ to $0.20, d-0.23$ to $-0.13, b-0.45$ to -0.34 ; goodness of fit: degrees of freedom $1352, R^{2} 0.35$, absolute sum of squares $264, s_{y \cdot x} 0.44$.

Statistical comparison demonstrated the superior performance of function 2 in both the whole data (Fig. 4a) and the subgroup of untreated subjects (Fig. 4b). Furthermore, the fitted curve obtained from data set 1 was readily transferable to data set 2 closely resembling the best fit in this data, as shown in Fig. 4a.

Subgroups of ambulatory or hospitalized patients and antibody-positive or -negative patients were also analyzed in data set 2 , with the result of function 2 providing a significantly better fit compared with function 1 (data not shown).

\section{Discussion}

The inverse relationship between TSH and $\mathrm{fT}_{4}$, created by negative thyroid pituitary hypothalamic feedback mechanisms, is central to our understanding of thyroid function and the clinical use of TSH determination for thyroid function testing. The relationship has been assumed to be of a log-linear nature $(1,2,9,11,13$, 14). When revisiting the relationship in the present study, our data did not lend support to the simple loglinear model. As a consequence, we propose a more complex and more accurate model based on the application of the erf formula.

The assumption of a simple linear relationship between $\log \mathrm{TSH}$ and $\mathrm{fT}_{4}$ was challenged by our initial observation that the correlation's gradient varied with thyroid function from the hypothyroid to the euthyroid and hyperthyroid range of data. We hypothesized that a model considering the extent of the deviation from a putative optimum set point might be more appropriate to represent the relationship between $\mathrm{fT}_{4}$ and $\mathrm{TSH}$ and, as a result, the alternate model was introduced for testing. In mathematical terms, the slope of the $\log \mathrm{TSH}-\mathrm{fT}_{4}$ relationship appears to vary with the distance from a mean $\mathrm{fT}_{4}$ value $\left((x-a)^{2}\right)$, and function 2 is derived as the integral of $d \log \mathrm{TSH} /$ $d \mathrm{fT}_{4}=k \mathrm{e}^{\left(-(q(x-a))^{2}\right)}+d$.

Further analyses and curve fitting confirmed that the application of the new function proved to be more appropriate to fit the data. The linear and new model were directly compared with the use of Prism, a powerful statistic software and curve fitting tool. On the basis of both $F$ statistics and Akaike criteria, which account for the natural tendency of a more complex model to yield a better fit $(12,15)$, the new model provided a significantly better fit. It should be noted that for the purpose of curve fitting with Prism, initial values of constants were derived from estimates based on the Excel data and SPPS plots.

The proposed new model was verified on a second independent set of data obtained from a later sampling 

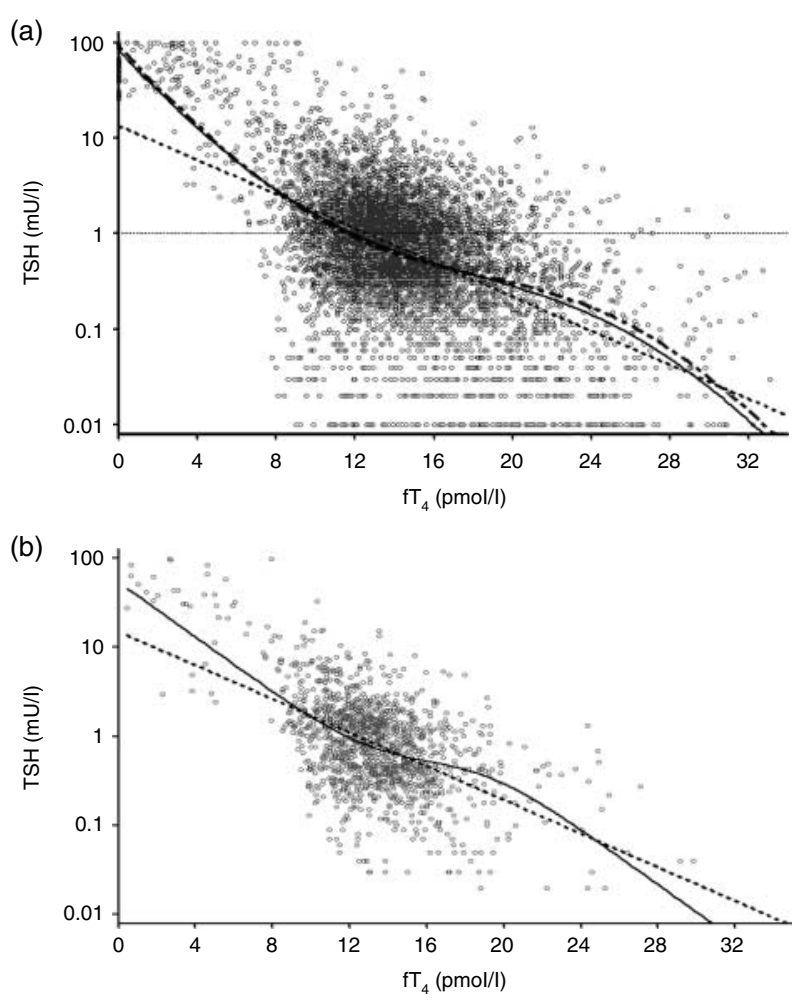

Figure 4 ( $a$ and $b)$ Application of the new model to data set 2. The curves and statistical results show a superior fit of function 2 (solid line) to data set 2 compared with the linear relationship of $\mathrm{fT}_{4}$ versus $\log$ TSH (function 1, discontinued line). For comparison, the fitted curve obtained from data set 1, Fig. 3 (dashed line), is also depicted in (a). Data were analyzed as a whole (a) and separately for untreated patients (b). Statistics are shown below, best-fit values under Results. Statistics for (a): probability null hypothesis function 1 is correct $0.01 \%$, alternative hypothesis function $299.99 \%$, preferred model function 2, differences in AICc 232.14, F:Dfn/Dfd 120.14/2.66, $P$ value $<0.0001$, conclusion $(\alpha=0.05)$ reject null hypothesis, $n=6605$. Statistics for (b): probability null hypothesis function 1 is correct $0.01 \%$, alternative hypothesis function 2 $99.99 \%$, preferred model function 2 , differences in AICc 45.22, F:Dfn/Dfd 25.00/2.13, $P$ value $<0.0001$, conclusion $(\alpha=0.05)$ reject null hypothesis, $n=1356$.

period. Again, analysis of total data and further subgroup evaluation in untreated patients unanimously favoured the complex function 2 over the straight line model. In addition, the fitted curve obtained from the first data was readily applicable to data set 2 .

The notion of an inverse log-linear function defining the relationship between $\mathrm{TSH}$ and $\mathrm{fT}_{4}$ in the feedback regulation of thyroid function was derived two decades ago from a limited number of experiments employing less refined assay techniques than are available today, superseding at that time the earlier idea of a direct linear relation $(1,2,9,10,16-18)$. While the log-linear function may still yield a rough estimate of the relation, it does not seem to provide a correct mathematical fit of the relationship. Based on the present data, we suggest that it should be replaced by a more refined model.
The biological interpretation of our refined model implies that the response of pituitary TSH depends on the deviation of circulating $\mathrm{fT}_{4}$ from an optimum set point and may turn out less vigorous and exaggerated as the magnitude of the deviation decreases.

The model may not apply to all situations, particularly in the case of interference due to hypothalamic pituitary failure or the presence of severe non-thyroidal illness affecting normal $\mathrm{TSH}_{-} \mathrm{fT}_{4}$ relation. Those patients had been excluded beforehand from the present study.

The possibility of a potential bias in $\mathrm{fT}_{4}$ immunoassays should be mentioned, but has been observed, almost exclusively, in specific populations such as pregnant women, patients with renal failure or severely ill patients that had been excluded (19-22). The overall performance of the $\mathrm{fT}_{4}$ assay has generally been good (22). Both accuracy and reliability of the assay have been ascertained by participation in inter-laboratory tests throughout the study period. Furthermore, separate analyses of ambulatory and hospitalized patients did not reveal any significant differences. Verification by an ultrafiltration method, considered the gold standard in $\mathrm{fT}_{4}$ measurement, was impractical with such a large sample size.

General limitations of a cross-sectional design apply to this study compared to a longitudinal study. In accordance with our findings, log-linear relationships were not always significant when assessed for each subject separately in a recent longitudinal observation on the assessment of the set point of the hypothalamuspituitary-thyroid axis in healthy volunteers by Benhadi et al. (10). In another earlier study, the slope of the InTSH/FTI relationship has been demonstrated to vary in patients with thyroid hormone resistance and to be characteristic of a specific mutation (23).

The present model focuses on describing the $\mathrm{TSH}-\mathrm{fT}_{4}$ relation. It does not investigate the diversity of mechanisms and multitude of parameters involved in pituitary thyroid regulation, such as short loop feedback, pulsatility of TSH secretion, stimulation of TSH by TRH and others that could be modelled from a cybernetic perspective $(24,25)$.

The proposed model of the $\mathrm{TSH}-\mathrm{fT}_{4}$ relationship, when refined by further studies, may be clinically useful to more precisely define subclinical states of thyroid dysfunction, particularly since the recent discussion on TSH reference range has cast doubt on TSH determination alone fulfilling this requirement (26-28). On the other hand, although the reference range may be a matter of debate, there has been strong evidence linking abnormal TSH in a patient to clinical disease, e.g. an increased probability of atrial fibrillation and an increased risk of mortality (29-31). Proposals of a combined consideration of TSH and thyroid hormones have so far failed to find broad acceptance $(32,33)$. A better understanding of the relationship between TSH and $\mathrm{fT}_{4}$ under various circumstances would 
facilitate a more individualized approach, based on the measurement of the two parameters, which might prove to be superior in judging risk of morbidity and mortality in a patient.

The present study demonstrates the complex nature of the $\mathrm{TSH}-\mathrm{fT}_{4}$ relation and should encourage other biological interrelations to be reconsidered in the light of the availability of better measurement techniques and the employment of more powerful computerized mathematical and statistical procedures.

\section{Declaration of interest}

The authors declare that there is no conflict of interest that could be perceived as prejudicing the impartiality of the research reported.

\section{Funding}

This research did not receive any specific grant from any funding agency in the public, commercial or not-for-profit sector.

\section{Acknowledgements}

The authors would like to thank Dr Ulrike Schulz, Institute for Medical Statistics, Medistat, Kiel, Germany for helpful discussions and advice in statistics.

\section{References}

1 Cohen JL. Thyroid stimulating hormone and its disorders. In Principles and Practice of Endocrinology and Metabolism, ch 20, pp 144-152. Eds KL Becker, JP Bilezikian, WJ Bremner \& W Hung. Philadelphia: Lippincott, Williams \& Wilkins, 1990.

2 Baloch Z, Carayon P, Conte-Devolx B, Demers LM, FeldtRasmussen U, Henry JF, LiVosli VA, Niccoli-Sire P, John R, Ruf J, Smyth PP, Spencer CA \& Stockigt JR. Guidelines Committee, National Academy of Clinical Biochemistry. Laboratory medicine practice guidelines. Laboratory support for the diagnosis and monitoring of thyroid disease. Thyroid 2003 13 3-126.

3 Biondi B \& Cooper D. The clinical significance of subclinical thyroid dysfunction. Endocrine Reviews 200829 76-131.

4 Surks M, Goswami G \& Daniels G. The thyrotropin reference range should remain unchanged. Journal of Clinical Endocrinology and Metabolism 200590 5489-5496.

5 Wartofsky L \& Dickey R. The evidence for a narrower thyrotropin reference range is compelling. Journal of Clinical Endocrinology and Metabolism 200590 5483-5488.

6 Brabant G, Beck-Peccoz P, Jarzab B, Laurberg P, Orgiazzi J, Szabolcs I, Weetman AP \& Wiersinga WM. Is there a need to redefine the upper normal limit of TSH? European Journal of Endocrinology 2006154 633-637.

7 Hollowell JG, Staehling NW, Flanders WD, Hannon WH, Gunter EW, Spencer CA \& Braverman LE. Serum TSH, $\mathrm{T}_{4}$, and thyroid antibodies in the United States population (1988 to 1994): National Health and Nutrition Examination Survey (NHANES III). Journal of Clinical Endocrinology and Metabolism $200287489-499$.

8 Voelzke H, Alte D, Kohlmann T, Luedemann J, Nauck M, John U \& Meng W. Reference intervals of serum thyroid function tests in a previously iodine-deficient area. Thyroid $200515279-285$.
9 Spencer C, LoPresti J, Patel A, Guttler R, Eigen A, Shen D, Gray D \& Nicoloff JT. Applications of a new chemiluminometric thyrotropin assay to subnormal measurement. Journal of Clinical Endocrinology and Metabolism $1990 \mathbf{7 0} 453-460$.

10 Carr D, McLeod DT, Parry G \& Thornes HM. Fine adjustment of thyroxine replacement dosage: comparison of the thyrotrophin releasing hormone test using a sensitive thyrotrophin assay with measurement of free thyroid hormones and clinical assessment. Clinical Endocrinology 198828 325-333.

11 Benhadi N, Fliers E, Visser TJ, Reitsma JB \& Wiersinga WM. Pilot study on the assessment of the setpoint of the hypothalamuspituitary-thyroid axis in healthy volunteers. European Journal of Endocrinology 2010162 323-329.

12 Motulsky H \& Christopoulos A. Fitting models to biological data using linear and nonlinear regression. A Practical Guide to Curve Fitting. New York: Oxford University Press, 2004.

13 Brabant G, Ocran K, Ranft U, von zur Muehlen A \& Hesch R. Physiological regulation of thyrotropin. Biochimie $1989 \mathbf{7 1}$ 293-301.

14 Demers L \& Spencer C. Thyrotropin/thyroid stimulating hormone (TSH) measurement. Thyroid 200313 33-44.

15 Akaike H. A new look at the statistical model identification. IEEE Transactions on Automatic Control 197419 716-723.

16 Crew FAE \& Wiesner BP. On the existence of a forth hormone, thyrotropic in nature, on the anterior pituitary. BMJ 19301 $777-778$.

17 Snyder P \& Utiger R. Inhibition of thyrotropin response to thyrotropin-releasing hormone by small quantities of thyroid hormones. Journal of Clinical Investigation 197251 2077-2084.

18 Spencer C, LoPresti J, Nicoloff J, Dlott R \& Schwarzbein D. Multiphasic thyrotropin responses to thyroid hormone administration in man. Journal of Clinical Endocrinology and Metabolism $199580854-859$.

19 Christofides ND, Wilkinson E, Stoddart M, Ray DC \& Beckett GJ. Serum thyroxine binding capacity-dependent bias in an automated free thyroxine assay. Journal of Immunoassay $1999 \mathbf{2 0}$ 201-221.

20 Saw S, Sethi S \& Aw TC. Technical evaluation of thyroid assays on the Vitros ECi. Clinical Chemistry 199945 578-580.

21 Sapin R. Serum thyroxine binding capacity-dependent bias in five free thyroxine immunoassays: assessment with serum dilution experiments and impact on diagnostic performance. Clinical Biochemistry 200134 367-371.

22 d'Herbomez M, Forzy G, Gasser F, Massart C, Beaudonnet A \& Sapin R. Clinical evaluation of nine free thyroxine assays: persistent problems in particular populations. Clinical Chemistry and Laboratory Medicine 200341 942-947.

23 Ercan-Fang S, Schwartz HL, Mariash CN \& Oppenheimer JH. Quantitative assessment of pituitary resistance to thyroid hormone from plots of the logarithm of thyrotropin versus serum free thyroxine index. Journal of Clinical Endocrinology and Metabolism 200085 2299-2303.

24 Dietrich JW, Tesche A, Pickardt CR \& Mitzdorf U. Thyrotropic feedback control: evidence for an additional ultrashort feedback loop from fractal analysis. Cybernetics and Systems 200435 315-331.

25 Fliers E, Unmehopa U \& Alkemade A. Functional neuroanatomy of thyroid hormone feedback in the human hypothalamus and pituitary gland. Molecular and Cellular Endocrinology 2006 $2511-8$

26 Ross D, Daniels G \& Gouveia D. The use and limitations of a chemiluminescent thyrotropin assay as a single thyroid function test in an out-patient endocrine clinic. Journal of Clinical Endocrinology and Metabolism 199071 764-769.

27 Andersen S, Bruun N, Pedersen K \& Laurberg P. Biologic variation is important for interpretation of thyroid function tests. Thyroid 200313 1069-1078.

28 Dickey R, Wartofsky L \& Feld S. Optimal thyrotropin level: normal ranges and reference intervals are not equivalent. Thyroid 2005 15 1035-1039. 
29 Sawin C, Geller A, Wolf P, Belanger A, Baker E, Bacharach P, Wilson PW, Benjamin EJ \& D'Agostino RB. Low serum thyrotropin concentrations as a risk factor for atrial fibrillation in older persons. New England Journal of Medicine 1994331 1249-1252.

30 Voelzke H, Schwahn C, Wallaschofski H \& Doerr M. Review: the association of thyroid dysfunction with all-cause and circulatory mortality: is there a causal relationship? Journal of Clinical Endocrinology and Metabolism 200792 2421-2429.

31 Ochs N, Auer R, Bauer D, Nanchen D, Gussekloo J, Cornuz J \& Rodondi N. Meta-analysis: subclinical thyroid dysfunction and the risk for coronary heart disease and mortality. Annals of Internal Medicine 2008148 832-845.
32 Meier C, Maisey M, Lowry A, Mueller J \& Smith M. Interindividual differences in the pituitary-thyroid axis influence the interpretation of thyroid function tests. Clinical Endocrinology 199339 101-107.

33 Falaschi P, Martocchia A, Proietti A, D’Urso R, Gargano S, Culasso F \& Rocco A. The hypothalamic-pituitary-thyroid axis in subjects with subclinical thyroid diseases: the impact of the negative feedback mechanism. Neuroendocrinology Letters 2004 25 292-296.

Received 9 March 2010

Accepted 17 March 2010 